\title{
Polyvagal and Global Cytokine Theory of Safety and Threat Covid-19 - Plan B
}

\author{
David Hanscom ${ }^{\mathrm{a}^{*}}$, David Roger Clawson ${ }^{\mathrm{b}}$, Stephen W. Porges ${ }^{\mathrm{c}}$, Ray Bunnage ${ }^{\mathrm{d}}$, \\ Les Aria ${ }^{\mathrm{e}}$, Steve Lederman ${ }^{\mathrm{f}}$, James Taylor ${ }^{\mathrm{g}}$, C. Sue Carter ${ }^{\mathrm{h}}$ \\ ${ }^{a}$ MD - Orthopedic Spine Surgeon, Oakland, CA, United States. \\ ${ }^{b}$ MD - Physical Medicine and Rehab, Seattle, WA, United States. \\ c PhD - "Distinguished University Scientist" Indiana Univ Bloomington, IN, United States. \\ ${ }^{d}$ Database and Visualization Developer, $U$ of WA Medicine, Seattle, WA, United States. \\ ${ }^{e}$ PhD - Pain psychologist, Kaiser Northern CA, Sacramento, CA, United States. \\ ${ }^{f} \mathrm{MD}$ - Cardiology, United States. \\ ${ }^{8}$ MD - Anesthesiologist, Pain physician, Pinehurst, NC, United States. \\ ${ }^{h}$ PhD - "Distinguished University Research Scientist", Kinsey Institute, Indiana University, Bloomington IN, United States. \\ Received 21 July 2020; Accepted 06 August 2020
}

\begin{abstract}
We are presenting this document to medical providers as a systematic approach to improve outcomes of patients with COVID-19. The following variables are considered: Autonomic nervous system viewed from the perspective of the Polyvagal Theory; Timing of interventions in terms of phase of the body's defense (Fight, Flight, Freeze, Faint); The nervous system considered the context of a "One System" perspective; Protein/Enzyme function; Immune system; Cytokine load - activity, inflammation and metabolic response; Viral load; Angiotensin 2 load.

The ARDS and multi-system organ failure of the COVID-19 is a complex problem. This approach acknowledges the complexity and presents a structure where the variables are systematically addressed.

1. The common risk factors for death are associated with baseline elevations of pro-inflammatory cytokines. Measures can be taken to lower them before being exposed to the virus-Plan A.

2. Strategies to optimize the body's defenses should be assessed and optimized. These include nutrition, vitamins, and trace elements, sleep, exercise, and minimizing threat.

3. The body's own resources are utilized through recruiting the autonomic nervous system to counteract elevated proinflammatory cytokines. The interventions are implemented in the context of what stage of defense the body is in-fight, flight, freeze, or faint.

4. Progressive pharmacological interventions are considered with the early interventions being those with minimal risk. We are asking the following:

- This approach is viewed as the foundation for clinical interventions. They should be implemented in a systematic and stepwise manner.

- Most of the treatments are already medically proven with minimal or no risk.

- All basic treatments are in place before more aggressive interventions are implemented.
\end{abstract}

\section{* Corresponding author: dnhanscom@gmail.com \\ dol http://dx.doi.org/10.28991/SciMedJ-2020-02-SI-2}

$>$ This is an open access article under the CC-BY license (https://creativecommons.org/licenses/by/4.0/).

(C) Authors retain all copyrights. 
- That this process be considered a framework to test clinical protocols and novel therapies. Much work needs to be done regarding dosing and timing.

- We are particularly interested in the potential of the following interventions, which do need to be looked at in a protocol. - Allowing ketosis in the Mid and Late Phases of the illness.

- Considering the use of ketone bodies instead of glucose for fuel in Mid and Late Phases of illness.

○ Eliminating glucocorticosteroids in the Early and Mid-Phases the use of steroids.

- Utilizing the anti-inflammatory cholinergic nervous system (vagal stimulation, nicotine patches, etc.).

○ Closer monitoring of IL-6 to in real time deliver the most appropriate interventions.

Keywords: Covid-19; Polyvagal Theory; Cytokine Theory; Coronavirus.

\section{The Protocol}

Covid-19 infections present a unique challenge to human physiology. In addition to activating our immune system the virus activates our inflammatory system in multiple ways, engaging autonomic reflexes bringing us ever closer to the edge of death if not death. There are four processes that are running in concert to create this stress on our bodies. The viral load, the cytokine load, the angiotensin load, and the dampened influence of vagal pathways on visceral organs and cytokine levels. Each of these needs to be mitigated to successfully negotiate a Covid-19 infection.

Our current societal response is to mitigate the viral load. We are distancing, isolating, masking, practicing good hygiene, trialing antiviral medications and trying desperately to bring on- successful vaccine on-line. There are multiple on-going trials to try and mitigate the cytokine load, but these receive little attention, let alone prioritization. These trials are mainly focusing on immunologic pharmaceutical interventions and not on more basic interventions including accessing and integrating our own innate recovery and healing responses. At this point we are unaware of a trial to specifically mitigate the angiotensin load.

There is so much more we could be doing. In this paper we have incorporated the Polyvagal Theory (Porges 1995, 2007, 2011 [1-3]) and the Global Cytokine Theory to provide a framework for how to better manage Covid-19 infections as well as other severe infections and associated shock syndromes. This paper will focus primarily on the most innate and physiologic strategies to help us survive a Covid-19 infection and secondarily on the use of pharmaceuticals to mitigate an escalating infection.

Let's explore these theories and strategies further.

\section{The Polyvagal Theory - Autonomic Nervous System Response}

As we struggle with the pandemic, we need to reinterpret and reframe our reactions within an informed appreciation of our nervous system - acknowledging that our reactions to the pandemic will only make sense if informed by an understanding of our evolution. Let's start with our physiologic and psychologic reactions to uncertainty and threat. We need to be able to regulate our physiologic state to move from the feeling of threat, fear and danger to a feeling of safety, security and trust in others. Functionally, the brain, visceral organs and fascial tissues are connected by neural pathways that send signals from the brain to our organs and tissues and from the organs and tissues to the brain. Consequently, threat reactions through definable and measurable pathways have predictable effects on our physical and mental health.

\section{“One-System” Perspective}

The term, "Mind-Body" is a misleading, even dangerous term. It implies that there is separation of the two. There is not; and one does not exist without the other. Living organisms respond to the environment as a unit. Humans have the additional characteristic of consciousness, which is manifested through language and thoughts. Research has demonstrated that ideas and concepts are embedded in our brain in the same manner as any other physical reality. It is one of the reasons that mental threats are processed in a similar way to physical ones [4].

Walter Hess in his 1949 Nobel Prize acceptance speech stated, "A recognized fact, which goes back to the earliest times is that every living organism is not the sum of a multitude of unitary processes, but is, by virtue of interrelationships and of higher and lower levels of control, an unbroken unity." This brief statement provides the context upon which development, application, and acceptance of neuro-autonomic disciplines, such as neurocardiology, neurogastroenterology, neuroendocrinology, neuropsychology, and neuroimmunology have emerged. This integrative "one-system" perspective encourages a better understanding of the dynamics of neural regulation of an integrated nervous system, yet we still see constraint and lack of integration by the limiting paradigms that are frequently used in the contemporary training and practice of medicine. 
We humans have a biological mandate of connectedness that requires a functional Social Engagement System (Porges 2009 [5]), which through common brainstem structures coordinate the muscles of the face, head and spine with the vagal regulation of the viscera and fascia. This system originates in a brainstem region known as nucleus ambiguus. Thus, the optimally resilient individual has opportunities to co-regulate their physiological state with a safe and trusted other. Ideally, this "other" person projects positive cues regarding their autonomic state through prosodic voice, warm welcoming facial expressions, gestures and posture of openness and accessibility. This allows vocalizations, facial expressions, gestures and postures modulated by autonomic states, to serve as cues of safety or threat to others. Together these pathways connect behavior to the nervous system and form the basis for social communication, cooperation, and connectedness.

\section{The Polyvagal Theory}

Polyvagal Theory, by articulating an evolutionary hierarchy in the function of the autonomic nervous system to challenges, provides a map of the state of the autonomic nervous system during any challenge. By understanding the autonomic state of an individual, this map informs us of the emergent behavioral, psychological and physiological reactivity that an individual may have in response to threat or alternatively to safe experiences.

The Polyvagal perspective helps us understand how the COVID-19 crisis moves us into physiological states of threat that disrupt our connectedness and places our physical and mental health at risk. We need to recognize opportunities to mitigate the potentially devastating reactions to threat, which in turn can destabilize the autonomic nervous system, resulting in visceral organ dysfunction and compromised physical and mental health. Awareness of the neural systems underlying Polyvagal Theory informs us that threats to survival can shift autonomic states, moving through sequential neural platforms that mimic evolution in reverse order, a process that John Hughlings Jackson labeled as dissolution [6]. Functionally, as we progress through a threat-induced trajectory of dissolution, we first lose the competence of our higher cognitive functions and the social engagement system. The uniquely mammalian myelinated vagal pathway originating in the brainstem area that also includes brainstem structures regulating vocal intonation, facial expressions, gestures, and postures to connect with others and calm our physiology goes off-line under threat. As well, the functions of our distinctly human neocortex show decreasing authority over our physiology, psychology and behavior. Without these resources, we move into adaptive, but more primitive defensive states.

\section{Fight /Flight to Freeze}

Our defense repertoire is first expressed as chronic mobilization, requiring activation of the pro-inflammatory cytokines and the sympathetic nervous system. Secondly, it expresses itself as immobilization, which is controlled by an evolutionarily older unmyelinated vagal pathway, the dorsal vagal pathway. In the absence of an active social engagement system, the mobilized state provides an efficient neural platform for fight and flight behaviors. If this state is activated for too long it will present as irritability, anger or chronic anxiety. When mobilization does not successfully move the individual into a safe context, then the nervous system shifts into an immobilized state with associated features of withdrawal, dissociation, syncope and death feigning. When this state becomes chronic it presents with loss of purpose, social isolation, despair, helplessness, hopelessness, depression, and at times psychosis.

Although both acute defensive strategies have adaptive values in protecting the individual, they are dependent on different neural pathways (i.e., high sympathetic tone or high dorsal vagal tone), which both interfere with interpersonal interactions, co-regulation, accessibility, trust, and feeling safe with another person. Higher level cognition and even language are also strategically and adaptively impaired. Thus, defensive states emerge, one aggressive to reactive, the other submissive to paralytic, from neural platforms that evolved to defend, while simultaneously compromising capacities to down-regulate our defenses through the co-regulation with a safe and trusted individual.

\section{What Autonomic State are You In?}

Basically, the theory emphasizes that in the presence of cues of predictable social interactions of support our nervous system of safety, the mammalian social engagement system, can down regulate our innate reactions to threat, whether the threat is tangible and observable or invisible, predicted or imagined. In this physiologic mode of safety, we have optimally high immunity and low inflammation. The case of Covid-19 is a battle between the virulence of the virus and the resilience of the system. Understanding these interactions is equivalent to calibrating risk on an individual level. With this information of risk, there is a better understanding of the foundational pathways through which the virus can have minor or deleterious consequences and thus lead to treatment that would minimize end-organ damage and death.

Polyvagal Theory informs us that autonomic state functions as an intervening variable moving the individual from states of vulnerability in response to threat to states of accessibility when supported by cues of safety and appropriate social support. Thus, knowing the physiological state of an individual provides a portal into understanding how they will respond to Covid-19 and the pandemic in its biopsychosocial totality. For example, if we are in an autonomic 
state of defense, the threat of illness will be compounded by the lack of opportunities to co-regulate. Thus, the public health strategies to flatten the curve and slow the transmission of the disease by social distancing and self-quarantining will exacerbate the negative impact that the virus and the pandemic will have on us.

\section{The Global Cytokine Theory within the Context of The Polyvagal Theory}

Global Cytokine Theory simply states that the cytokine system is the fundamental communicator within our bodies and brains having major governance over our physiologic and psychologic states and our behaviors. The "proinflammatory" cytokines dictate the threat state. The "anti-inflammatory" cytokines dictate the safe state. The cytokine system is much more than an adjunct of the immune system and controls much more than just inflammation. The cytokine system is a mediator of the Polyvagal states and integrates neurocardiology, neurogastroenterology, neuroendocrinology, neuroimmunology, neuropsychology, and neurosociology. The cytokine system is the fundamental communicator via intracellular transmission, neural transmission and/or hormonal like transmission for the body and the brain that results in the dissolution of any separation of systems - let alone the mind-body duality.

\section{Threat}

The "pro-inflammatory" cytokines, threat cytokines, are the underpinnings of the majority of our chronic physical illness and diseases. They are the underpinnings of $100 \%$ of our mental illness and diseases, including addictions. Also, they are certainly elevated in all who suffer social illness and disease (isolation, crowding, poverty, disenfranchisement, discrimination and injustice) [7].

With elevations in our "pro-inflammatory" cytokines we see the progression from fight to flight to freeze to faint and the associated physiology, psychology and behaviors of these states. Under the influence of the "proinflammatory" cytokines we become catabolic, asexual, disconnected, selfish, reactive and irritable, and in the extreme dissociated or even in a state of collapse. At this end point we have very poor immunity.

This has direct implications for the primary reason we are dying from Covid 19 - cytokine "storms".

\section{Safety}

On the other hand, the "anti-inflammatory" cytokines, safety cytokines, are the underpinnings of health and wellness. With the elevations of the "anti-inflammatory" cytokines we see the states of breed and feed and digest and rest. It is here where we are anabolic, restorative, connected, bonded, sexual, reproductive, cognitive and creative. We, also, have high immunity.

In addition to influencing our immune status and promoting inflammation in the body the "pro-inflammatory" cytokines also dictate our metabolism through very complex pathways. Suffice it to say that when we are in fight, flight, and fever we favor glucose for fuel in the battle. However, when we move into freeze, faint, and collapse we favor ketones for fuel. Glucose reserves and gluconeogenesis may be falling in advanced attacks and therefore another energy source is required, but, also, the ketones act as a brake on the "pro-inflammatory" cytokines to decrease their production and also serve to protect critical tissues such as neurons, nephrons and both cardiac and skeletal myocytes.

"Pro-inflammatory" cytokines are fuel producing through breakdown of tissues in fight, flight and fever. They are catabolic. Tumor necrosing factor was first known as cachectin. Prolonged elevation of "pro-inflammatory" cytokines leads to tissue and organ compromise.

\section{Combined Approach to Methodically Treat COVID-19}

The Polyvagal Theory and the Global Cytokine Theory have direct implications in the management of Covid-19 and its associated pandemic. Let's look at this crisis through this lens to see how we might optimally manage our health and well-being and defeat this devastating virus.

\section{Viral Load}

Our primary strategy today is mitigating the viral load. The practices of distancing, isolating, masking and precise hygiene have been the primary way of controlling this virus. Other strategies to mitigate viral load are antiviral medications that can block the replication in an infected person. Beyond that we are holding on to the promise of a vaccine to protect us from a Covid-19 infection. There are multiple issues with just focusing on the viral load. Distancing, isolating and hygiene do not develop immunity. We are just hiding from the virus, but when we come out of hiding the virus is still there. Months of this strategy has protected health care systems from a crushing onslaught of patients and a potential system collapse, but has also caused social and financial trauma, and by the University of Washington estimates we still have a very low percent of the population that has been exposed to the virus. This makes it very difficult to come out of hiding and infection rates will just rise upon exposure to the virus. We need about $60-70 \%$ exposure and immunity to significantly decrease the risk of coming out of hiding. When this level is achieved, the virus just doesn't have enough viable vectors to propagate itself. We are a long way away from this level of herd immunity and barely getting closer with our current strategy. To complicate this more the length of time we 
can carry antibodies and immunity may be very short. Within months of illness we may be again at risk for infection. We don't know if a vaccine will provide sustained and prolonged immunity. Finally, the state of health in the US is so poor with associated high-risk comorbidities that it may be difficult for us to achieve herd immunity through exposure and illness as the risk of tissue and organ injury and death is too high. Our lack of investment in this area has come home to roost.

We can hope that antiviral medications are going to be successful. However, the track record for antivirals is less than stellar and they do not enhance immunity. So, we wait for a vaccine while life has come to a halt. A vaccine is months to years away and even if one is developed the efficacy may be poor or immunity may be limited.

On top of these issues this virus has a propensity to mutate and today's vaccine or antiviral may not be the one that works tomorrow. Mitigating the viral load may be a constantly moving target. A vaccine is part of a solution but certainly not the most important part of the solution and certainly a vaccine shouldn't be nearly the sole focus in this crisis. In fact, mitigating the viral load shouldn't be the main focus either.

\section{Cytokine Load}

People are not being disabled or dying from the viral load. They are dying from cytokine loads primarily. Certainly, the viral load plays a significant part in creating a "cytokine storm", but there are many other factors in someone's total cytokine load that place them at risk for death.

Cytokine load is a combination of the viral cytokine load + the person's pre-existing cytokine load from their preexisting medical conditions and nutritional status (see above) + the person's cytokine load from their pre-existing chronic as well as acute mental health conditions + the person's cytokine load from their pre-existing social and financial stressors - yes, living in isolation, poverty, disenfranchisement, discrimination and injustice elevates people's cytokine load and places them at increased risk to "storm" with a Covid-19 infection.

Note that all these risk factors shift the regulation of our physiological state into a defensive mode at great expense to the support and regulation of the homeostatic processes that support health, growth, and restoration. The immune system in partnership with other regulatory circuits including endocrine and autonomic systems shift their functional strategies from support of homeostasis to defense when dealing with the viral threat. Certainly, the immune system isn't running wild as immunity is falling during these "storms". Inflammation seems to be running wild or "storming", but this may actual represent a relatively normal viral cytokine load in a person with a high baseline cytokine load that pushes them over a threshold into collapse, organ failure and death.

Mitigating cytokine loads needs to be the primary focus of stopping the destruction and devastation of this virus. If we learn to successfully mitigate cytokine loads the viral infection becomes a relative non-event. Plus, successfully mitigating cytokine loads will be available to us for all future pandemics, epidemics, and similar shock syndromes. It will not change or mutate like a virus. This is where the solution lies for returning life to normal.

Two plans or approaches mitigate the "pro-inflammatory cytokine" load that are equally important will be outlined.

- Plan A - Lower the baseline/pre-exposure pro I cytokine load

- Plan B - Minimize the impact with a multi-pronged approach after exposure**

\section{Angiotensin Load}

The cytokine load is really the knockout punch in this fight, but there is another process that makes Corona viruses so dangerous and deadly and that is angiotensin - specifically angiotensin 2. Angiotensin as you may guess by its name helps support our blood pressure when we are under attack. It is part of our fight and flight response. It is present when we are under attack whether by a person, an animal or a parasite, bacteria or virus. The "pro-inflammatory" cytokine response plays a role in activation the vasoconstricting and inflammatory angiotensin system - they are tightly related [8].

Angiotensin 2 is not only highly vasoconstrictive, but it is also highly inflammatory. We have an enzyme in our body called angiotensin converting enzyme 2 (ACE2) that under normal conditions would cleave angiotensin 2 into vasodilatory and anti-inflammatory angiotensins. Angiotensins flow freely through our blood stream and throughout our bodies. ACE2 exists in our blood and on the surface of our cells making up our blood vessels as well as other our tissues and organs. We have a high number of ACE2 receptors lining our blood vessels, in our nose, on our tongues, in our throats, and on the cells of all our organs - brain, lungs, heart, kidneys, gonads and other organs.

Here is the problem: the Covid-19 virus skewers these receptors with its spike proteins to attach to our cells and then enter our cells through these receptor sites to highjack the mechanics of our cells for replication and then

\footnotetext{
* Acknowledging that the "pro-inflammatory" cytokines are a necessary part of defending threats including infections; therefore, Plan B has a critical timing phase that identifies when the "pro-inflammatory" cytokine response needs to be mitigated or blunted and some interventions prior to this point could result in poorer outcomes.
} 
distribution. To attack this virus our immune system must also attack tissues where the virus attaches (ACE2 receptors) and there can be collateral damage in this attack. To make this situation worse the occupation and destruction of ACE2 leaves the highly vasoconstrictive and highly inflammatory angiotensin 2 in our blood and tissues for far too long at too high of a concentration. This can cause inflammation of blood vessels and organs, clots and infarctions in our limbs and organs including the brain (strokes) and heart (attacks).

In addition, angiotensin 2 inhibits the part of our nervous system located in our brainstem that communicates with our bodies, especially our organs and endothelial tissues lining our blood vessels, through our vagus nerves that deescalates the inflammatory cytokine response in favor of and anti-inflammatory response. Angiotensin 2 thus does its own inflammatory damage, but, also, perpetuates and complicates a cytokine storm. This response needs mitigation along with the mitigation of the cytokine and viral loads.

\section{Overview of Interventions and Timing}

We are most interested in the most basic strategies that should improve outcomes with the least risk of doing harm within the management of a Covid-19 infection. To manage an infection well it is important to understand the changing physiology of the different phases of infections. With Covid-19 five phases are important to recognize:

- Pre-exposure - see Plan A

- Exposure - no symptoms

- Early infection - fight, fever

- Mid infection - flight -> freeze

- Late infection - faint, collapse

We encourage proactively doing things to enhance immunity and decrease "pro-inflammatory" cytokine load in the Pre-exposure phase and to maintain these interventions throughout an illness - see Plan A.

We feel it is important to allow the Early Infection Phase - fight, fever - to run for at least 24 hours other than perhaps the introduction of a safe and effective antiviral medication. This allows our body's innate immune system to fight off the virus, drop the viral load and shed the virus from our systems. Aggressive Mid-phase interventions could blunt this early and necessary immune response. The majority of people with Covid-19 infections will defeat the virus in this Early-Infection Phase.

The Mid-Infection Phase signals a change or transition in our innate physiology and strategy to not only fight the virus but to protect ourselves. Note the inflection point * on the graph below. This transition is marked by a downregulation in metabolism and interestingly cellular immunity, too. It is in this window that the system is starting to fatigue and this phase is marked by escalating inflammation and falling immunity. In this window, temperature, metabolism, heart rate and blood pressure will start to fall and our natural physiology will move from glucose to ketones for fuel. Ketones and ketosis act as feedback system to depress "pro-inflammatory" cytokines and also are protective to critical cells and cellular functions in a low metabolic, low perfusion and low oxygenation states. It is important to not interfere but to support this phase of the infection and our innate strategy to defeat the virus.

Therefore, we contend when we move into this phase it is important to avoid aggressive pressor (proinflammatory) interventions and glucose support including the use of glucocorticosteroids. We believe that glucocorticosteroids are a stress hormone to promote fuel for the Early-Phase of infection. The glucocorticosteroids do have other effects including some ability to support sodium retention and blood pressure, and they are able to inhibit "pro-inflammatory" cytokines, but their prolonged use leads to falling immunity, excessive catabolism with tissue and organ deterioration and prevents the protective effects of ketones and ketosis [9].

We believe there are multiple physiologic markers to monitor to determine when the physiologic state is moving from the Early-Phase to the Mid-phase. As this changing physiology is mediated by "pro-inflammatory" cytokines we feel that point of care testing of Interleukin 6 may be the most accurate data point to help understand this shift and the timing of interventions. When the system is fatiguing in the fight and we are transitioning from the Early-Phase to the Mid-Phase we feel aggressive pressor support becomes counterproductive and contraindicated. We feel glucocorticosteroids should be withheld throughout the course of Covid-19 in favor of deployment of antiinflammatory, anabolic and pro-immunity strategies, particularly activation of the vagal anti-inflammatory cholinergic system. By prevention of further exhaustion of the system and mitigation of the "pro-inflammatory" cytokine load at the Mid-Phase of infection we believe the Late-Phase of faint, collapse, tissue destruction, organ failure and death can be avoided. 


\section{Polyvagal Strategies Beginning with Suspected Illness/ Early-Phase}

Many basic, do no harm, strategies are available for mitigating the "pro-inflammatory" cytokine load outlined below, and let's focus first on the Polyvagal strategies.

There are many resources within Polyvagal Theory that trigger endogenous anti-inflammatory/anabolic actions through vagal efferent activity. Think of vagal mediated anti-inflammatory processes as performing a 'negative' feedback mechanism maintaining cytokine levels within an optimal or homeostatic range that would optimize health and preparation in anticipation of threat. Polyvagal Theory proposes, based on well documented research, that vagal efferents from the nucleus ambiguus actively dampen sympathetic and inflammatory reactions to threat. Thus, social connectedness and cues of safety, which shift the autonomic nervous system out of a state of defense into a state that supports feelings of safety will be accompanied by increases in vagal efferent function that will support homeostatic processes such as health, growth, and restoration. Since there is a substantial literature documenting the role of vagal efferents and acetylcholine (the neurotransmitter being used by the vagus) in dampening "pro-inflammatory"/catabolic cytokines and enhancing and distributing "anti-inflammatory"/anabolic cytokines (see the research by Tracey 2002 [10]), plus the recent plausible hypotheses that COVID-19 is selectively attacking cholinergic systems, several noninvasive techniques can be implemented.

\section{Vagal Stimulation}

Stimulation of vagal, trigeminal, and facial afferents on the external ear and face (e.g., forehead) communicate with the brainstem nuclei, including the nucleus ambiguus, regulating the vagus and provide a validated technology to stimulate vagal function. In addition, other noninvasive devices can improve vagal function through electrical stimulation of the neck.

- Since modulated vocalizations trigger calming pathways that output through the vagus, it may be possible to use a noncontact 'ambient' acoustic vagal nerve stimulator [11]. This noncontact stimulator would only require playing modified vocal music. that emphasized the modulation of acoustic frequencies similar to a mother's lullaby.

- Can be done simply with washcloth to forehead or acupuncture to ear lobe.

- This can be accomplished by computer processing vocal music to exaggerate the intonation variations that our nervous system detects as cues of safety. The algorithms modifying the vocal music functionally amplify the positive prosodic information, which would effectively and efficiently calm the autonomic nervous system by increasing vagal efferent outflow to downregulate sympathetic excitation and pro-inflammatory cytokine load. Metaphorically, this would be similar to a mother's voice or lullaby calming a crying baby.

\section{Safety}

Another key factor in mitigating inflammation is feeling safe even in the midst of a severe threat such as COVID-19 and possible death. Every measure should be taken to ensure the patient feels safe, seen and secure. This strategy is based on a well-document neurophysiological pathway in which vagal function is enhanced while being calm and feeling safe and secure.

- Safe and soothing environment;

- Fully listening;

- Clear communication with what is going on;

- Pleasant soothing sounds - earphones with relaxing music;

- Pleasant somatic sensations - light touch, massage;

- Pleasant smells - the smell of certain plant oils can reduce "pro-inflammatory" cytokines;

- Pleasant tastes - good food with spices that reduce "pro-inflammatory" cytokines are preferred to salt or sugar for flavour.

\section{Socialization}

Another factor that promotes health and feeling safe is socialization. It is how the human race evolved and also why the ventral complex of the vagus nerve is in proximity of the facial and postural muscles innervations. Safe socialization down-regulates the fight and flight response and "pro-inflammatory" cytokines. Connection with others is critical to our survival $[12,13]$.

- Educate the family as to the need to stay connected; 
- Facetime/Zoom meetings;

- With precautions allow the family to interact with the patient;

- Have the patient stay up to date on family activities and include virtually if possible;

- OT/ PT/SW/Behavioral Health services;

- Online interactive games.

\section{Breath/ Relaxation Techniques}

\section{The Science of Breathing}

Research shows that when we are under stress (i.e., real or perceived), the brain and body are dominated by stress chemicals, and prolonged exposure results in alterations of the body's homeostasis and can complicate the pathophysiological conditions of acute or chronic diseases (Chovatiya and Medzhitov 2014 [14]). This compensatory response to stress is known as the stress responses (fight-flight-or-freeze), which is the function of the sympathetic nervous system (SNS).

Hence, if the SNS, which is often referred to as the physiological gas pedal, is dominant for a prolong period of time, its effects are pronounced by structural changes in the brain and on the nervous system [15-17]. The counterbalance to the ANS is the Parasympathetic Nervous System (PNS), the physiological brake pedal. This is a built-in evolutionary system to help bring us back to homeostasis once the threat is resolved and thus permitting the body's state to engage in reparative and restorative processes, a relaxation response [18, 19].

Research suggest we can induce this relaxation response of the PNS through voluntary slow breathing exercises (e.g., diaphragmatic breathing and alternate nostril breathing) (Brown et al. 2005 [20]; Jerath et al. 2006 [21]; Naik et al. 2018 [22]; Telles et al. 2017 [23]) and applications of mindfulness practices [24]. Activation of the relaxation response helps stimulate the vagus nerve, which is measured via-a-vis respiratory sinus arrhythmia (RSA). Our heart rate increases in inhalation (SNS) and decreases on the exhalation of the breath [25].

The RSA is an indicator of the vagal tone. Higher vagal tone means the body relaxes faster after stress. The greater the amplitude of RSA, the greater the vagal tone [26]. Hence, engaging in slow breathing exercises during stressful experiences (mental or physical) at a rate of 6-10 breaths per minutes helps optimize ventilation gas, arterial oxygenation, gas exchange and arterial oxygenation helps maximize vagal tone, and thereby stimulating the PNS [2732].

Given the evidence and benefits of slow breathing exercises on health and well-being, and decreased mortality in diseased states (Aeschbacher et al. 2016 [33]; Radaelli et al. 2004 [26]; Tsuji 1996 [34]), it is surprising that the medical community has not prescribed this effective and simple tool part as part of a standard care of medical treatment for those struggling with acute and chronic diseases. Slow breathing exercises are a vital part of optimizing health.

There are many choices of breathing techniques that upregulate the ventral vagal tone. Here are some examples.

- Slow exhalations especially linked with deep short inhalations may enhance the vagal efferent action through the ventral vagal pathways to the sino-atrial node. Respiration functions as a gating mechanism turning on and off the vagal efferent flow [35, 36];

- Relaxed slow breathing;

- Alternate nostril breathing.

There are numerous other relaxation strategies. At least one should be thoughtfully considered if dealing with a COVID-19 infection.

- Mindfulness/meditation;

- Biofeedback/ medical hypnosis;

- Visualization;

- Slow-movement martial arts.

Support of the vagal, "anti-inflammatory" cholinergic, system decreases inflammation and increases immunity. This system should not be ignored in patient care. It is our anabolic and healing response. Substituting glucocorticosteroid's anti-inflammatory effect for this system is felt to be a mistake and stunts the recovery and healing process. 
The vagal system is the primary focus in this treatment paradigm, but there are other benign supportive measures outlined below that should be employed, too.

\section{Sleep}

Restorative sleep is essential to recovery, healing and homeostasis. It is as essential as social connection to the human species. A single night of lost sleep will be associated with a rise in "pro-inflammatory" cytokines and recovery sleep is associated with a decrease in "pro-inflammatory" cytokines and an elevation in "anti-inflammatory" cytokines. Whether in the preventative phase of infection (see Plan A) through the later phases of infection attention to sleep is imperative. This can be challenging when in the hospital and particularly when in an ICU with excessive activity, lighting, noises and interruptions. Maintenance of circadian rhythms and attention to sleep hygiene needs to be practiced in all settings for optimal recover, restoration, healing and health.

\section{Inclusive Monitoring and Treatment Considerations}

It is critical to continue with the Polyvagal strategies throughout the progression of disease-especially if the situation becomes serious.

\section{Home}

There are a number of things we can do at home if we think we are coming down with a Covid-19 infection:

\section{Monitor}

- Temperature - home thermometer;

- Oxygenation - home finger oximeter;

- Heart rate variability - home device;

- (Monitoring specific components of heart rate variability (i.e., respiratory sinus arrhythmia) that are valid indices of vagal regulation of the heart through pathways originating in nucleus ambiguus can be of additional value.).

\section{Intake}

- Maintain fluids;

- Maintain fuel;

○ Whole unprocessed foods;

o Simple carbohydrates are ok - need glucose for the fever and the fight;

$\circ$ Basic anti-inflammatory and immune support;

○ Vitamin D 5,000 units daily [37];

○ Vitamin K2 1000 ug daily [38];

o Vitamin C 500 mg PO q8 [39];

○ Vitamin B complex daily

- Magnesium $400 \mathrm{mg}$ PO q daily [40];

o Zinc 5 mg PO q8 [41-43];

○ Melatonin 5 mg PO q pm;

- Lecithin (contains fatty acid phosphatidylcholine a precursor of acetylcholine);

○ Aspirin $81 \mathrm{mg}$ daily to prevent vascular inflammation and clotting;

○ Sleep - > 7 hours/ night.

If feeling short of breath or if oxygen saturations or HRV are falling then seek medical attention.

\section{Institutional care - Early-Phase}

\section{Monitor}

Labs

- Complete blood count with differential;

- Basic metabolic panel, Magnesium, Zinc;

- CRP, Lactate, Procalcitonin, Cytokine Panel* [44]; 
- Prothrombin time, Fibrinogen, D dimer!

- Albumin [45].

Imaging

- Chest X-ray;

- Chest CT scan.

Vitals**

- Temperature;

- Oxygenation;

- Heart rate;

- Heart rate variability;

- Blood pressure;

- Respiratory rate.

*If CRP, Lactate, Interleukin 6 are showing aggressive upward trends then the patient is starting Mid Phase physiology - flight -> freeze.

***If these vital signs are falling then the patient may be starting into Mid Phase physiology - flight -> freeze.

If Prothrombin Time, Fibrinogen, D dimer are abnormal then the patient may be already into Late Phase physiology - faint, collapse - activate shock protocol

\section{Initial}

- Supportive care;

- Oxygen via nasal cannula;

- Continue Home recommendations;

- Polyvagal modalities;

- If albumin $<3.6$ then aggressive nutritional supplementation/ dietary consult.

\section{Medications}

- Antivirals may be appropriate here;

- Interferons may also have a role here.

If remaining stable in Early-Phase physiology - fight, fever, high fuel - then simple carbohydrates, glucose are ok need glucose for fever and fight.

If showing signs of instability - see below - then stop simple carbohydrates and activate the Mid-Phase strategies. *Supplemental glucocorticosteroids have no role [46].

\section{Fighting the Storm - Mid Phase - The Critical Point in Care}

In the Mid Phase, the ultimate goal is to prevent the "pro-inflammatory" cytokines from crossing a threshold that pushes the system into the Late Phase physiology of faint $->$ collapse -> organ failure -> death. It is at this inflection point $(*)$ where it is necessary to mitigate the cytokine load more aggressively even if it delays the shedding of the viral load (Figure 1). 


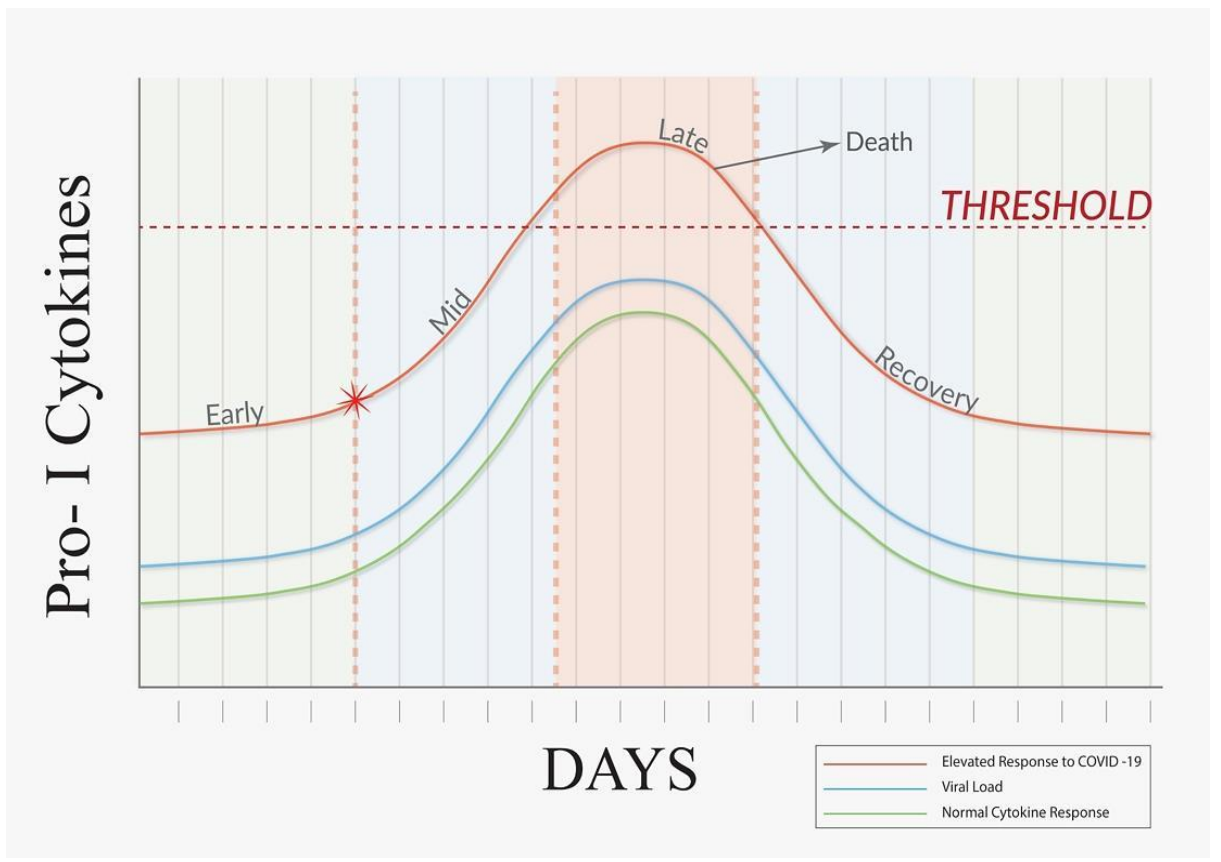

Figure 1. The Critical Point in Care (Graph by Dr. Clawson, MD)

Most of the interventions including allowing ketosis are specific to shutting down the pro-inflammatory cytokine storm [9]. Ketosis can also slow viral replication. Ketosis will protect vital cells in a state of low perfusion, low oxygenation and low metabolism.

\section{Initial Basic Interventions}

- Vitamin D 50,000 units PO now and $\mathrm{q}$ am $\mathrm{x} 3$ days [37];

- Vitamin K2 1000 ug PO now and q am x 3 days [38];

- Vitamin C $1500 \mathrm{mg}$ IV now and q8 [39];

- MgSO4 1000 mg IV now and q8 [40];

- Zinc $5 \mathrm{mg}$ IV or PO po now and q8 (if low then replace to keep plasma levels normal - do not super supplement - need a normal value for enzymes to deactivate pro inflammatory angiotensins and the cytokines);

- DHEA $5 \mathrm{mg}$ PO now and q pm [7];

- Melatonin $5 \mathrm{mg}$ PO now and q pm;

- Vitamin B pack IV now and q pm;

- Fuel: Avoid dextrose/glucose/simple carbohydrates [7].

○ PO: high quality fats rich in omega3 fatty acids, medium chain triglycerides and high quality proteins [56];

○ EN: high quality fats rich in omega3 fatty acids, medium chain triglycerides, small peptides);

○ PN: lipids (high quality anti-inflammatory lipid emulsion rich in omega3 fatty acids and medium chain triglycerides) and amino acids (rich in leucine, lysine, isoleucine, threonine, tryptophan, tyrosine and phenylalanine), no dextrose with these) IV now and $q$ am.

- Anticlotting treatment: Heparins SQ;

- Sleep.

*Continue Polyvagal modalities

*Avoid pressors/adrenergics if possible

- Low dose dobutamine, dopamine and norepinephrine for support are preferred;

- Epinephrine and vasopressin are much more pro-inflammatory and should be avoided;

- If ketosis is allowed then lower MAPs will be better tolerated.

*No glucose or glucocorticosteroids - allow ketosis [46]

Although there are a number of markers for trending the physiologic decline towards shock, or faint and collapse, physiology, however we feel IL6 is likely the most precise marker therefore we recommend rechecking IL6 stat hourly - if IL6 is still climbing then more aggressive strategies should be employed to mitigate the cytokine load. 
Advancing Interventions

Antibiotics - tetracyclines have antibacterial, anti-viral and anti-cytokine activity. They are also neuroprotective.

- Minocycline 200 mg IV now and 100 mg q12 after or [47];

- (Azithromycin is not preferred due to potential cardiac toxicity);

Gabanergics decrease 'pro-inflammatory" cytokines and control fear and anxiety

- Gabapentin 100-300 mg PO q8

Cholinergics mimic the effects of the vagal system and decrease "pro-inflammatory" cytokines and increase "antiinflammatory" cytokines and cellular immunity

- Urecholine $10 \mathrm{mg}$ po $\mathrm{q} 8$

- Nicotine 3.5 - $7 \mathrm{mg}$ patch now and daily [48]

Hormonals from the sex/anabolic categories decrease "pro-inflammatory" cytokines, promote calm, trust and a sense of safety, and promote cellular immunity and tissue repair

- DHEA 25-50 mg now and q pm [7]

- Oxytocin is anti-inflammatory and may be of value and enhance recovery. It is elevated when a patient feels safe and cared for.

We suggest the above interventions prior to more aggressive interventions that may have more worrisome side effects through the disruption and inhibition of normal immune functions. However, if the "pro-inflammatory" cytokines are continuing to climb and are approaching a critical level where the patient may fall into frank shock then more aggressive immunologic and biologics seem to be indicated. Using these interventions too early could result in poorer outcomes if immunity is blunted too soon. We suggest rechecking IL6 stat - hourly - if still climbing then advance.

\section{Interleukin inhibition - IL 6}

- Tocilizumab $400 \mathrm{mg}$ IV

Pro-inflammatory cytokine inhibitors are potent focal blockers of a specific cytokine and its functions to decrease the "proinflammatory cytokine" load/activity and can mitigate a cytokine storm. IL6 seems to be the most proinflammatory of the group and therefore is the preferred target, but other "pro-inflammatory" cytokine inhibitors have been used with reported success. IL6 inhibition may increase the risk for bacterial infections or other complications from associated immune dysfunction.

\section{Other Considerations}

If Mid-Phase mitigation fails and the patient is progressing into Late-Phase physiology we would consider more aggressive inotropic and vasopressor support as seen in traditional shock protocols appropriate but would still feel that glucocorticosteroids would only be useful as an extreme rescue measure (Keller et al. 2020 [49]) and could potentially be harmful by reversing the anti-inflammatory and protective effects of ketones and ketosis.

Again, these considerations are made in the light of trying to mitigate the progression to Late-Phase physiology particularly at the inflection point just prior to the Mid-Phase of the infection. It is our feeling that the Mid-Phase is under-recognized and this results in lost opportunities to mitigate the cytokine load prior to the system progressing into shock with impaired immunity, high inflammation, tissue and organ injury and potential death.

Here is a more complete list of potential interventions worthy of consideration to mitigate a Covid-19 infection and its progression to a shock syndrome and death:

\section{Interventions that Mitigate Viral Load}

\section{PHYSICAL FACTORS}

Distancing

- Prevents transmission

Isolating

- Prevents transmission

Hygiene

- Prevent transmission 
Masking

- Prevents transmission

Vaccines

- Prevents infection by priming the immune system to the viral proteins thus destroying the virus prior to invading the cells and replicating

Herd Immunity

- Prevents sustained transmission

\section{MEDICAL}

Early Phase

Antiviral medications - remdesivir,

Interferons - interferon alpha-2b, gamma

- Activate anti-viral immune system

- Prevents replication

Human recombinant ACE2*

- Binds the virus in the blood where it is destroyed prior to attaching to or invading the cells and replicating

\section{Human recombinant $A C E 2 *$}

- Binds the virus in the blood where it is destroyed prior to attaching to or invading the cells and replicating

- Interferons

- Activate anti-viral immune system

\section{Treatments that Mitigate Cytokine Load}

\section{VITAMINS AND SUPPLEMENTS}

Vitamin D [37]

- Reduces pro-inflammatory cytokines

Vitamin Bs [50]

- Reduces pro inflammatory cytokines

Vitamin C [39]

- Reduces pro-inflammatory cytokines and may have anti-viral properties

Vitamin K [38]

- Reduces inflammation

Astaxanthine

- Reduces pro inflammatory cytokines

Quercetin

- Reduces pro inflammatory cytokines and may have antiviral properties

Glutathione/N Acetyl Cysteine [51]

- Reduces pro inflammatory cytokines, oxidative stress and may have anti-viral properties

Alpha lipoid acid [52]

- Reduces pro inflammatory cytokines

\section{GABA}

- Reduces pro inflammatory cytokines and anxiety

Melatonin [53-54]

- Reduces pro inflammatory cytokines

Alpha tocopherols

- Reduces pro inflammatory cytokines

Phosphatidylcholine

- Reduces pro inflammatory cytokines and raises anti-inflammatory cytokines

\section{TRACE ELEMENTS}

Magnesium [40, 55] 
- Reduces pro inflammatory cytokines and angiotensin 2; factor in Vit D function

Zinc [41-43]

- Key in enzymes that cleave pro inflammatory cytokines and may have anti-viral properties

DIET/NUTRITION/METABOLIC

Anti-inflammatory dietary oils (fish oil, avocado oil, walnut oil, etc.)

- Reduces pro inflammatory cytokines

Anti-inflammatory spices (turmeric, cinnamon, clove, ginger etc.)

- Reduces pro inflammatory cytokines

Anti-inflammatory oils (eucalyptus, lavender, rosemary, tea tree) Licorice root

- Reduces pro inflammatory cytokines

Licorice root

- Reduces pro inflammatory cytokines and may have anti-viral and anti-bacterial properties. Supports blood pressure.

Ketosis - in Mid-Late Phases

- Deprives system of glucose needed for viral replication

Reduces pro inflammatory cytokines (Pinto)

Ketogenic diet (Pinto)

- Reduces pro-inflammatory cytokines and is neuroprotective

Ketone bodies (recommend beta-hydroxybutyrate over acetoacetate or acetone)

- Reduce pro inflammatory cytokines

\section{MEDICATIONS}

Benzodiazepines

- Reduces pro inflammatory cytokines and anxiety

Gabapentin [57]

- Reduces pro inflammatory cytokines and anxiety

Cannabinoids [58-60]

- Reduce pro inflammatory cytokines and anxiety

Urecholine

- Reduces pro inflammatory cytokines and raises anti-inflammatory cytokines

Nicotine (patch) [48]

- Reduces pro inflammatory cytokines and raises anti-inflammatory cytokines

Tetracyclines

- Reduce pro inflammatory cytokines, antibacterial and some antiviral properties

Azithromycin (Meyer et al. 2009 [61]) (cardiotoxicity is a risk)

- Reduces pro inflammatory cytokines, antibacterial and some antiviral properties

Glucophage

- Reduces pro inflammatory cytokines - decreases insulin resistance

Statins

- Reduce pro inflammatory cytokines - risk for mitochondrial dysfunction and myopathy

\section{Other}

Interleukin 1 inhibitor

Interleukin 6 inhibitor

Interleukin 17 inhibitor

Tumor Necrosing Factor inhibitor

Interferon alpha- $2 b$

Interferon gamma

Interleukin 10 
Interleukin 4

Alpha 7 acetylcholine receptor agonist

Phosphodiesterase Inhibitors

- Reduce pro inflammatory cytokines

Histamine inhibitors

- Reduce pro inflammatory histamines and anxiety

Tyrosine kinase inhibitors - Acalabrutinib (Roschewski) [62]

- Selective inhibitor of Bruton Tyrosine Kinase (BTK)

IV Immunoglobulin

- Reduces pro inflammatory cytokines

Oxytocin [62]

- Reduces pro-inflammatory cytokines, increases anti-inflammatory cytokines, raises ventral vagal tone, facilitates GABA relaxation, analgesic, promotes safety, and promotes anabolism and healing.

Anabolic hormones (DHEA, testosterone, estrogen, progesterone, pregnanolone) [7]

- Reduce pro inflammatory cytokines and promote recovery and healing

Angiotensin 2 lowering strategies

- See angiotensin below

\section{Upregulating Ventral Vagus}

Vagal stimulation (touch, social, electrical, acoustical) [10]

- Reduces pro inflammatory cytokines and raise anti-inflammatory cytokines

Breath control exercises

- Reduce pro inflammatory cytokines and raise anti-inflammatory cytokines

\section{Mitigating Angiotensin 2 And Associated Effects}

\section{Human recombinant ACE2*}

Converts angiotensin 2 to angiotensin 1-7 thus disinhibiting vagal tone and reducing inflammation/ catabolism

ACE inhibitors

- Prevent the formation of angiotensin 2, drops inflammation, but may leave more unoccupied ACE2 for viral attachment, and side of cough and edema via persistent bradykinin

Angiotensin receptor blockers

- Blocks effects of angiotensin 2, drops inflammation, but may leave more circulating angiotensin 2

*Noted the potential role of human recombinant ACE2 in mitigated viral, cytokine and angiotensin 2 loads.

\section{Other Interventions}

Antiplatelet medications

- Reduce inflammation and clotting

Anticoagulants

- Reduce clotting

\section{Conclusion}

In summary, we feel there is a lack of recognition of some of our innate physiologic strategies that help us fight and survive viral infections, but most specifically the sequelae of high cytokine loads. We have outlined the role of Polyvagal Theory and Global Cytokine Theory in Safety and Threat for consideration in the treatment of Covid-19 infections. We have outlined the critical Mid-Phase of the course of infection that requires phase specific and precisely timed interventions to prevent the deterioration into Late-Phase physiology where things become much more perilous and it is difficult to execute a successful rescue. The premise is to start treatment with the most physiologic and benign strategies - at first do no harm - and progress to more aggressive strategies as the situation becomes more dire. We feel there are substantial interventions available to reduce viral, cytokine and angiotensin 2 loads, and their deleterious 
effects, to prevent the progression to collapse, tissue destruction, organ failure and death. It is with this in mind that we have offered up these considerations and hope this will lead to further discussions, research and optimal care for Covid-19 and other severe infections and shock syndromes.

\section{Background Information}

\section{CYTOKINES}

Cytokines phylogenetically are nearly a billion years old and are small bits of protein ( 100-200s amino acid sequences) that allow communication between cells. They are the major factor in the expression of the immune system in both stimulating it (inflammatory) or down-regulating it (anti-inflammatory). There are cytokine receptors on the surface of every cell in the body. They also act as neurotransmitters, are key in hormonal expression and regulate metabolism. They are numerous and have been classified by their source of production, target of action, effect, connection with disease states. For the focus of this discussion they will simply be categorized by warding off of threat (pro-inflammatory) or promoting recovery and regeneration (anti-inflammatory). This is Table 1 from the paper written by Foster and Samman 2012 [41]).

\section{CYTOKINES}

\section{Pro-inflammatory}

Primary - IL1 beta, IL6, TNF alpha

Others - IL2, 15, 17, 18

\section{Anti-inflammatory}

Primary - IL 4, 10, 4, 13, TGF beta

Others - IL37, 38

Table 1. Selected Cytokines. Cell sources and examples of their principal functions in inflammation (Foster and Samman [41])

\begin{tabular}{|c|c|c|}
\hline Cytokine & Primary Cell Sources & Key Functions in Inflammation \\
\hline IL-1 & Macrophages Endothelial cells & $\begin{array}{l}\text { Synthesis of acute phase proteins by hepatocytes; Local and systemic } \\
\text { inflammatory effects }\end{array}$ \\
\hline IL-2 & Activated T cells Th1 cells & Proliferation of T cells, B cells; Proliferation and activation of NK cells \\
\hline IL-6 & $\begin{array}{l}\text { Macrophages Th2 cells Endothelial cells } \\
\text { Adipocytes Myocytes Osteoblasts }\end{array}$ & $\begin{array}{l}\text { Synthesis of acute phase proteins by hepatocytes; Proliferation of B cells; } \\
\text { Down-regulation of IL-1 and TNF production; Activation of immune cells, } \\
\text { osteoclasts, endothelial cells; Hypothalamic Pituitary Axis-fever \& } \\
\text { hormone release }\end{array}$ \\
\hline IL-10 & MacrophagesTh 2 cells & $\begin{array}{l}\text { Resolution of inflammation; Inhibition of Th1 inflammatory cytokine } \\
\text { synthesis; Inhibition of activated macrophages and dendritic cells }\end{array}$ \\
\hline IL-12 & Macrophages Dendritic cells & $\begin{array}{l}\text { Promotion of Th1 differentiation; Stimulation of IFN- } \gamma \text { production by T cells, } \\
\text { NK cells }\end{array}$ \\
\hline TNF- $\alpha$ & $\begin{array}{l}\text { Macrophages T cells NK cells Lymphoid } \\
\text { cells Endothelial cells Adipocytes Cardiac } \\
\text { myocytes Fibroblasts Neuronal cells }\end{array}$ & $\begin{array}{l}\text { Synthesis of acute phase proteins by hepatocytes; Recruitment and activation } \\
\text { of neutrophils and monocytes at sites of infection; Stimulation of CRP release } \\
\text { from liver; Activation of NF- } \mathrm{kB} \text { pathway; Induction of insulin resistance }\end{array}$ \\
\hline TGF- $\beta$ & Macrophages T cells & $\begin{array}{l}\text { Resolution of inflammation; Limit production of IL-2, IFN- } \gamma \text {, and TNF; } \\
\text { Inhibition of proliferation/activation of B cells, T cells, macrophages. }\end{array}$ \\
\hline IFN- $\gamma$ & Th1 cells NK cells & $\begin{array}{l}\text { Activation of macrophages; Suppression of Th2 cell activity; Promotion of } \\
\text { leukocyte migration }\end{array}$ \\
\hline
\end{tabular}

Abbreviations: IFN, interferon; IL, interleukin; NK, natural killer; NF-kB, nuclear factor-kappaB; Th, T helper;

TGF, transforming growth factor; TNF, tumour necrosis factor $[64,65]$.

\section{Acknowledgements}

The team acknowledges the remarkable insights provided by Dr. Clawson and Stephen Porges. The basis of this paper arose from the combination of their in-depth knowledge of the immune system, inflammatory response, cell biology, metabolism, autonomic nervous system, neuroimaging, and neurophysiology.

This document evolved out of this group's efforts to further the treatment of chronic pain. It became apparent that the potential solution to the COVID-19 virus is similar to that of solving chronic pain. That is defining all of the relevant variables in a given patient and addressing them simultaneously. 


\section{Declaration of Competing Interest}

The authors declare that they have no known competing financial interests or personal relationships that could have appeared to influence the work reported in this paper.

\section{Ethical Approval}

All procedures performed in studies involving human participants were in accordance with the ethical standards of the institutional and/or national research committee and with the 1964 Helsinki declaration and its later amendments or comparable ethical standards.

\section{References}

[1] PORGES, S. W. (1995). Orienting in a defensive world: Mammalian modifications of our evolutionary heritage. A Polyvagal Theory. Psychophysiology, 32(4), 301-318. doi:10.1111/j.1469-8986.1995.tb01213.x.

[2] Porges, S. W. (2007). The polyvagal perspective. Biological Psychology, 74(2), 116-143. doi:10.1016/j.biopsycho.2006.06.009.

[3] Porges, S. W. (2011). The polyvagal theory: neurophysiological foundations of emotions, attachment, communication, and selfregulation (Norton Series on Interpersonal Neurobiology). WW Norton \& Company.

[4] Barrett, L. F. (2014). The Conceptual Act Theory: A Précis. Emotion Review, 6(4), 292-297. doi:10.1177/1754073914534479.

[5] Porges, S. (2009). Polyvagal Theory 1: Basic Principles (Phylogny, Neoception, Dissolution, Social Engagement System). Lecture recorded at NSW Service for the Treatment and Rehabilitation of Torture and Trauma Survivors, New South Wales.

[6] Jackson, J. H. (1884). The Croonian Lectures on Evolution and Dissolution of the Nervous System. BMJ, 1(1215), 703-707. doi:10.1136/bmj.1.1215.703.

[7] Heffner, Kathi L. "Neuroendocrine Effects of Stress on Immunity in the Elderly: Implications for Inflammatory Disease." Immunology and Allergy Clinics of North America 31, no. 1 (February 2011): 95-108. doi:10.1016/j.iac.2010.09.005.

[8] Zambelli, V., Grassi, A., \& Bellani, G. (2012). Role of the Renin-Angiotensin System in ARDS. Annual Update in Intensive Care and Emergency Medicine 2012, 171-181. doi:10.1007/978-3-642-25716-2_17.

[9] Youm, Y.-H., Nguyen, K. Y., Grant, R. W., Goldberg, E. L., Bodogai, M., Kim, D., ... Dixit, V. D. (2015). The ketone metabolite $\beta$-hydroxybutyrate blocks NLRP3 inflammasome-mediated inflammatory disease. Nature Medicine, 21(3), 263269. doi:10.1038/nm.3804.

[10] Tracey, K. J. (2002). The inflammatory reflex. Nature, 420(6917), 853-859. doi:10.1038/nature01321.

[11] Porges, S. W., \& Dana, D. A. (2018). Clinical Applications of the Polyvagal Theory: The Emergence of Polyvagal-Informed Therapies (Norton Series on Interpersonal Neurobiology). WW Norton \& Company.

[12] Porges SW. (2020) The COVID-19 Pandemic is a paradoxical challenge to our nervous system: a Polyvagal Perspective. Clin Neuropsychiatry 17, 135-8. doi: 10.36131/CN20200220.

[13] Porges, SW. (2020). U.S. Patent No. 10,661,046. Washington, DC: U.S. Patent and Trademark Office.

[14] Chovatiya, R., \& Medzhitov, R. (2014). Stress, Inflammation, and Defense of Homeostasis. Molecular Cell, 54(2), 281-288. doi:10.1016/j.molcel.2014.03.030.

[15] Kiecolt-Glaser, J. K., and Glaser, R. (1991). Stress and Immune Function in Humans. Psychoneuroimmunology, $849-867$. doi:10.1016/b978-0-12-043780-1.50038-5.

[16] Reiche, E. M. V., Nunes, S. O. V., \& Morimoto, H. K. (2004). Stress, depression, the immune system, and cancer. The Lancet Oncology, 5(10), 617-625. doi:10.1016/s1470-2045(04)01597-9.

[17] Reznikov, L. R., Grillo, C. A., Piroli, G. G., Pasumarthi, R. K., Reagan, L. P., \& Fadel, J. (2007). Acute stress-mediated increases in extracellular glutamate levels in the rat amygdala: differential effects of antidepressant treatment. European Journal of Neuroscience, 25(10), 3109-3114. doi:10.1111/j.1460-9568.2007.05560.x.

[18] Nakao, M. (2019). Special series on "effects of board games on health education and promotion" board games as a promising tool for health promotion: a review of recent literature. BioPsychoSocial medicine, 13(1), 5. doi:10.1186/s13030-019-0146-3.

[19] Nakao, M. (2019). Heart Rate Variability and Perceived Stress as Measurements of Relaxation Response. Journal of Clinical Medicine, 8(10), 1704. doi:10.3390/jcm8101704.

[20] Brown, Richard P., and Patricia L. Gerbarg. "Sudarshan Kriya Yogic Breathing in the Treatment of Stress, Anxiety, and Depression: Part I-Neurophysiologic Model." The Journal of Alternative and Complementary Medicine 11, no. 1 (February 2005): 189-201. doi:10.1089/acm.2005.11.189.

[21] Jerath, R., Edry, J. W., Barnes, V. A., \& Jerath, V. (2006). Physiology of long pranayamic breathing: Neural respiratory elements may provide a mechanism that explains how slow deep breathing shifts the autonomic nervous system. Medical Hypotheses, 67(3), 566-571. doi:10.1016/j.mehy.2006.02.042. 
[22] Naik, G. S., Gaur, G. S., \& Pal, G. K. (2018). Effect of modified slow breathing exercise on perceived stress and basal cardiovascular parameters. International journal of yoga, 11(1), 53. doi:10.4103/ijoy.IJOY_41_16

[23] Telles, S., Gupta, R. K., Yadav, A., Pathak, S., \& Balkrishna, A. (2017). Hemisphere specific EEG related to alternate nostril yoga breathing. BMC Research Notes, 10(1). doi:10.1186/s13104-017-2625-6.

[24] Chin, M. S., \& Kales, S. N. (2019). Understanding mind-body disciplines: A pilot study of paced breathing and dynamic muscle contraction on autonomic nervous system reactivity. Stress and Health, 35(4), 542-548. doi:10.1002/smi.2887.

[25] Gerritsen, R. J. S., \& Band, G. P. H. (2018). Breath of Life: The Respiratory Vagal Stimulation Model of Contemplative Activity. Frontiers in Human Neuroscience, 12. doi:10.3389/fnhum.2018.00397.

[26] Radaelli, A., Raco, R., Perfetti, P., Viola, A., Azzellino, A., Signorini, M. G., \& Ferrari, A. U. (2004). Effects of slow, controlled breathing on baroreceptor control of heart rate and blood pressure in healthy men. Journal of Hypertension, 22(7), 1361-1370. doi:10.1097/01.hjh.0000125446.28861.51.

[27] Bilo, G., Revera, M., Bussotti, M., Bonacina, D., Styczkiewicz, K., Caldara, G., .. Parati, G. (2012). Effects of Slow Deep Breathing at High Altitude on Oxygen Saturation, Pulmonary and Systemic Hemodynamics. PLoS ONE, 7(11), e49074. doi:10.1371/journal.pone.0049074.

[28] Byeon, K., Choi, J.-O., Yang, J. H., Sung, J., Park, S. W., Oh, J. K., \& Hong, K. P. (2012). The Response of the Vena Cava to Abdominal Breathing. The Journal of Alternative and Complementary Medicine, 18(2), 153-157. doi:10.1089/acm.2010.0656.

[29] Dick, T. E., Mims, J. R., Hsieh, Y.-H., Morris, K. F., \& Wehrwein, E. A. (2014). Increased cardio-respiratory coupling evoked by slow deep breathing can persist in normal humans. Respiratory Physiology \& Neurobiology, 204, 99-111. doi:10.1016/j.resp.2014.09.013.

[30] Giardino, N. D., Glenny, R. W., Borson, S., \& Chan, L. (2003). Respiratory sinus arrhythmia is associated with efficiency of pulmonary gas exchange in healthy humans. American Journal of Physiology-Heart and Circulatory Physiology, 284(5), H1585-H1591. doi:10.1152/ajpheart.00893.2002.

[31] Hayano, J., Yasuma, F., Okada, A., Mukai, S., \& Fujinami, T. (1996). Respiratory Sinus Arrhythmia. Circulation, 94(4), 842847. doi:10.1161/01.cir.94.4.842.

[32] Ito, S., Sasano, H., Sasano, N., Hayano, J., Fisher, J. A., \& Katsuya, H. (2006). Vagal nerve activity contributes to improve the efficiency of pulmonary gas exchange in hypoxic humans. Experimental Physiology, 91(5), 935-941. doi:10.1113/expphysiol.2006.034421.

[33] Aeschbacher, S., Schoen, T., Dörig, L., Kreuzmann, R., Neuhauser, C., Schmidt-Trucksäss, A., ... Conen, D. (2016). Heart rate, heart rate variability and inflammatory biomarkers among young and healthy adults. Annals of Medicine, 49(1), 32-41. doi:10.1080/07853890.2016.1226512.

[34] Tsuji, J. (1996). Innovations in organic synthesis. Angewandte Chemie-German Edition, 108(18), 2291.

[35] Eckberg, D. L. (2003). The human respiratory gate. The Journal of Physiology, 548(2), 339-352. doi:10.1113/jphysiol.2002.037192.

[36] Hering, H. E. (1910). A functional test of heart vagi in man. Menschen Munchen Medizinische Wochenschrift, 57, $1931-1933$.

[37] Ilie, P. C., Stefanescu, S., \& Smith, L. (2020). The role of vitamin D in the prevention of coronavirus disease 2019 infection and mortality. Aging Clinical and Experimental Research, 32(7), 1195-1198. doi:10.1007/s40520-020-01570-8.

[38] Harshman, S. G., \& Shea, M. K. (2016). The Role of Vitamin K in Chronic Aging Diseases: Inflammation, Cardiovascular Disease, and Osteoarthritis. Current Nutrition Reports, 5(2), 90-98. doi:10.1007/s13668-016-0162-x.

[39] Kashiouris, M. G., L'Heureux, M., Cable, C. A., Fisher, B. J., Leichtle, S. W., \& Fowler, A. A. (2020). The Emerging Role of Vitamin C as a Treatment for Sepsis. Nutrients, 12(2), 292. doi:10.3390/nu12020292.

[40] Sugimoto, J., Romani, A. M., Valentin-Torres, A. M., Luciano, A. A., Ramirez Kitchen, C. M., Funderburg, N., ... Bernstein, H. B. (2012). Magnesium Decreases Inflammatory Cytokine Production: A Novel Innate Immunomodulatory Mechanism. The Journal of Immunology, 188(12), 6338-6346. doi:10.4049/jimmunol.1101765.

[41] Foster, M., \& Samman, S. (2012). Zinc and Regulation of Inflammatory Cytokines: Implications for Cardiometabolic Disease. Nutrients, 4(7), 676-694. doi:10.3390/nu4070676.

[42] Prasad, A. S., Beck, F. W., Bao, B., Fitzgerald, J. T., Snell, D. C., Steinberg, J. D., \& Cardozo, L. J. (2007). Zinc supplementation decreases incidence of infections in the elderly: effect of zinc on generation of cytokines and oxidative stress. The American Journal of Clinical Nutrition, 85(3), 837-844. doi:10.1093/ajcn/85.3.837.

[43] Cabrera, Á. J. R. (2015). Zinc, aging, and immunosenescence: an overview. Pathobiology of Aging \& Age-Related Diseases, 5(1), 25592. doi:10.3402/pba.v5.25592.

[44] Neghaiwi, BH., 2020, Roche test receives FDA emergency use approval for COVID-19 patients. Reuters Available online: https://www.reuters.com/article/us-roche-hldg-elecsys/roche-test-receives-fda-emergency-use-approval-for-covid-19-patientsidUSKBN23B0IC (accessed on 3 June 2020).

[45] Gibbs, J. (1999). Preoperative Serum Albumin Level as a Predictor of Operative Mortality and Morbidity. Archives of Surgery, 134(1), 36. doi:10.1001/archsurg.134.1.36.

[46] Bernard, G. R., Luce, J. M., Sprung, C. L., Rinaldo, J. E., Tate, R. M., Sibbald, W. J., ... Brigham, K. L. (1987). High-Dose Corticosteroids in Patients with the Adult Respiratory Distress Syndrome. New England Journal of Medicine, 317(25), 15651570. doi:10.1056/nejm198712173172504. 
[47] Krady, J. K., Basu, A., Allen, C. M., Xu, Y., LaNoue, K. F., Gardner, T. W., \& Levison, S. W. (2005). Minocycline Reduces Proinflammatory Cytokine Expression, Microglial Activation, and Caspase-3 Activation in a Rodent Model of Diabetic Retinopathy. Diabetes, 54(5), 1559-1565. doi:10.2337/diabetes.54.5.1559.

[48] Piao, W.-H., Campagnolo, D., Dayao, C., Lukas, R. J., Wu, J., \& Shi, F.-D. (2009). Nicotine and inflammatory neurological disorders. Acta Pharmacologica Sinica, 30(6), 715-722. doi:10.1038/aps.2009.67.

[49] Keller, M. J., Kitsis, E. A., Arora, S., Chen, J.-T., Agarwal, S., Ross, M. J., ... Southern, W. (2020). Effect of Systemic Glucocorticoids on Mortality or Mechanical Ventilation in Patients With COVID-19. Journal of Hospital Medicine, 15(8), 489-493. doi:10.12788/jhm.3497.

[50] Mikkelsen, K., Stojanovska, L., Prakash, M., \& Apostolopoulos, V. (2017). The effects of vitamin B on the immune/cytokine network and their involvement in depression. Maturitas, 96, 58-71. doi:10.1016/j.maturitas.2016.11.012.

[51] Horowitz, R. I., Freeman, P. R., \& Bruzzese, J. (2020). Efficacy of glutathione therapy in relieving dyspnea associated with COVID-19 pneumonia: A report of 2 cases. Respiratory Medicine Case Reports, 30, 101063. doi:10.1016/j.rmcr.2020.101063.

[52] Akbari, M., Ostadmohammadi, V., Tabrizi, R., Mobini, M., Lankarani, K. B., Moosazadeh, M., ... Asemi, Z. (2018). The effects of alpha-lipoic acid supplementation on inflammatory markers among patients with metabolic syndrome and related disorders: a systematic review and meta-analysis of randomized controlled trials. Nutrition \& Metabolism, 15(1). doi:10.1186/s12986-018-0274-y.

[53] Sánchez-López, A. L., Ortiz, G. G., Pacheco-Moises, F. P., Mireles-Ramírez, M. A., Bitzer-Quintero, O. K., Delgado-Lara, D. L. C., ... Velázquez-Brizuela, I. E. (2018). Efficacy of Melatonin on Serum Pro-inflammatory Cytokines and Oxidative Stress Markers in Relapsing Remitting Multiple Sclerosis. Archives of Medical Research, 49(6), 391-398. doi:10.1016/j.arcmed.2018.12.004.

[54] Hardeland, R. (2019). Aging, Melatonin, and the Pro- and Anti-Inflammatory Networks. International Journal of Molecular Sciences, 20(5), 1223. doi:10.3390/ijms20051223.

[55] Deng, X., Song, Y., Manson, J. E., Signorello, L. B., Zhang, S. M., Shrubsole, M. J., ... Dai, Q. (2013). Magnesium, vitamin D status and mortality: results from US National Health and Nutrition Examination Survey (NHANES) 2001 to 2006 and NHANES III. BMC Medicine, 11(1). doi:10.1186/1741-7015-11-187.

[56] Pinto, A., Bonucci, A., Maggi, E., Corsi, M., \& Businaro, R. (2018). Anti-Oxidant and Anti-Inflammatory Activity of Ketogenic Diet: New Perspectives for Neuroprotection in Alzheimer's Disease. Antioxidants, 7(5), 63. doi:10.3390/antiox7050063.

[57] Yamaguchi, K., Kumakura, S., Someya, A., Iseki, M., Inada, E., \& Nagaoka, I. (2017). Anti-inflammatory actions of gabapentin and pregabalin on the substance P-induced mitogen-activated protein kinase activation in U373 MG human glioblastoma astrocytoma cells. Molecular Medicine Reports, 16(5), 6109-6115. doi:10.3892/mmr.2017.7368.

[58] Papagianni, E. P., \& Stevenson, C. W. (2019). Cannabinoid Regulation of Fear and Anxiety: an Update. Current Psychiatry Reports, 21(6). doi:10.1007/s11920-019-1026-z.

[59] Liu, C., Ma, H., Slitt, A. L., \& Seeram, N. P. (2020). Inhibitory Effect of Cannabidiol on the Activation of NLRP3 Inflammasome Is Associated with Its Modulation of the P2X7 Receptor in Human Monocytes. Journal of Natural Products. doi:10.1021/acs.jnatprod.0c00138.s001.

[60] Tanasescu, R., \& Constantinescu, C. S. (2010). Cannabinoids and the immune system: An overview. Immunobiology, 215(8), 588-597. doi:10.1016/j.imbio.2009.12.005.

[61] Meyer, M., Huaux, F., Gavilanes, X., van den Brûle, S., Lebecque, P., Lo Re, S., ... Leal, T. (2009). Azithromycin Reduces Exaggerated Cytokine Production by M1 Alveolar Macrophages in Cystic Fibrosis. American Journal of Respiratory Cell and Molecular Biology, 41(5), 590-602. doi:10.1165/rcmb.2008-0155oc.

[62] Roschewski, M., Lionakis, M. S., Sharman, J. P., Roswarski, J., Goy, A., Monticelli, M. A., ... Wilson, W. H. (2020). Inhibition of Bruton tyrosine kinase in patients with severe COVID-19. Science Immunology, 5(48), eabd0110. doi:10.1126/sciimmunol.abd0110.

[63] Diep, P. T., Buemann, B., Uvnäs-Moberg, K., \& Marazziti, D. (2020). Oxytocin, a possible treatment for COVID-19? Everything to gain, nothing to lose. Clinical Neuropsychiatry, 17(3). doi:10.36131/CNEPUB20201703.

[64] Abbas, A.K.; Lichtman, A.H. (2005) Cellular and Molecular Immunology, 5th ed.; Elsevier Saunders: Philadelphia, PA, USA.

[65] Zhou, X., Fragala, M. S., McElhaney, J. E., \& Kuchel, G. A. (2010). Conceptual and methodological issues relevant to cytokine and inflammatory marker measurements in clinical research. Current Opinion in Clinical Nutrition and Metabolic Care, 13(5), 541-547. doi:10.1097/mco.0b013e32833cf3bc. 